\section{P763 ATTENUATION OF SYPHILIS INFECTION FOLLOWING IMMUNIZATION OF RABBITS WITH A TRIVALENT ANTIGEN COCKTAIL}

${ }^{1}$ Barbara Molini* ${ }^{2}$ Charmie Godornes, ${ }^{2}$ Maria Partida-Aguilar, ${ }^{2}$ Austin Haynes, ${ }^{3}$ Alloysius Gomez, ${ }^{4}$ Darrick Carter, ${ }^{1}$ Lorenzo Giacani, ${ }^{3}$ Caroline Cameron, ${ }^{2}$ Sheila Lukehart. ${ }^{1}$ University of Washington - Harborview Medical Center, Medicine - Allergy and Infectious Diseases, Seattle, USA; ${ }^{2}$ University of Washington, Medicine, Seattle, USA; ${ }^{3}$ University of Victoria, Biochemistry and Microbiology, Victoria, Canada; ${ }^{4}$ University of Washington, Global Health, Seattle, USA

\subsection{6/sextrans-2019-sti.821}

Background The bacterium that causes syphilis, Treponema pallidum subsp. pallidum (Tp), elicits cellular and humoral immune responses to numerous antigens during infection. We have identified three recombinant peptide antigens that, when used separately for immunization, show promise in the attenuation of chancre development or dissemination to distant tissues. Here, we report protection induced by a three-antigen cocktail emulsified in either of two custom adjuvants containing Natural or Synthetic TLR4 agonists + a natural Mincle agonist.

Methods Three purified recombinant peptides [TprK (aa37273), Tp0751 (aa24-237), and Tpr Subfamily I (23-351)] were emulsified in either adjuvant and used to immunize groups of 8 rabbits. The immunized rabbits and 8 Unimmunized controls were challenged intradermally with $10^{\wedge} \mathrm{Tp} /$ site at 10 sites. Lesion development was recorded daily. Treponemal burden was measured by darkfield (DF) microscopy and qPCR of lesion aspirates, and dissemination to distant tissues was evaluated by rabbit infectivity test (RIT).

Results Compared to Unimmunized, treponemal burden by DF in lesion aspirates at Day 19 was lower in both Natural $(\mathrm{P}=0.001)$ and Synthetic $(\mathrm{P}=0.004)$ groups; by qPCR, treponemal burden was lower in the Natural group $(\mathrm{P}=0.008)$. At Days 19 and 30, the proportion of lesions ulcerating was lower in the Natural group, compared to Unimmunized $(\mathrm{P}=0.0001$ [d.19] and $\mathrm{P}=0.0002,[\mathrm{~d} .30])$. At day 30, the proportion of lesions ulcerating in the Natural group was lower than in the Synthetic $(\mathrm{P}=0.04)$ group. Mean lesion volume was smaller in immunized groups versus Unimmunized on days 10-25. RIT indicated the lowest number of disseminated $T p$ in rabbit tissues from the Natural group, followed by the Synthetic group, then the Unimmunized group ( $\mathrm{P}=0.0247)$.

Conclusion Immunization with the three-antigen cocktail significantly attenuates syphilis infection: enhancing $T p$ clearance, promoting lesion healing, and reducing dissemination. In rabbits, Natural adjuvant was more effective than Synthetic adjuvant in inducing protective immunity.

Disclosure No significant relationships.

\section{P764 GETTING TO THE BOTTOM OF IT: SEXUAL POSITIONING AND SYPHILIS STAGE AT DIAGNOSIS AMONG MEN WHO HAVE SEX WITH MEN}

\footnotetext{
${ }^{1}$ Vincent Cornelisse*, ${ }^{2}$ Eric Chow, ${ }^{1}$ Rosie Latimer, ${ }^{3}$ Janet Towns, ${ }^{2}$ Marcus Chen, ${ }^{2}$ Catriona Bradshaw, ${ }^{3}$ Christopher Fairley. 'Melbourne Sexual Health Centre, Carlton, Australia; ${ }^{2}$ Alfred Health, Melbourne Sexual Health Centre, Carlton, Australia; ${ }^{3}$ Melbourne Sexual Health Centre, Melbourne, Australia
}

10.1136/sextrans-2019-sti.822
Background Effective syphilis control could be achieved by reducing its duration of infectiousness, for example, by identifying a higher proportion of cases at the primary stage. We hypothesised that men who have sex with men (MSM) who practice receptive anal intercourse ("bottoms") are more likely to miss the primary stage and present with secondary syphilis, compared to MSM who practice exclusively insertive anal intercourse ("tops").

Methods This was a retrospective analysis of MSM diagnosed with either primary or secondary syphilis at Melbourne Sexual Health Centre between 2008 and 2017. We analysed associations between the stage of syphilis (primary vs secondary) and sexual behaviour data collected by computer-assisted self-interview (CASI).

Results 559 MSM diagnosed with syphilis provided sufficient behavioural data for analysis, of whom 338 (60\%) had primary syphilis and 221 (40\%) had secondary syphilis. Among "tops", 77\% (95\%CI 69-84) presented with primary syphilis and 23\% (95\%CI 16-31) presented with secondary syphilis. Whereas among "bottoms", 54\% (95\%CI 49-59) presented with primary syphilis and 46\% (95\%CI 41-51) presented with secondary syphilis. Among those with primary syphilis, 247 $(73 \%, 95 \% \mathrm{CI} 68-78)$ had a penile chancre and $77(23 \%$, 95\%CI 19-28) had an anal chancre. In multivariate logistic regression, "bottoms" were more likely to present with secondary syphilis than "tops" (aOR 3.90, p<0.001), after adjusting for age, HIV status, and condom use.

Conclusion "Bottoms" more often presented with secondary syphilis compared to "tops", and most MSM who presented with primary syphilis had penile chancres rather than anal chancres. This suggests that MSM who have receptive anal intercourse may be more likely to overlook anal syphilis chancres, perhaps because these are located inside their anal canal. These men may benefit from additional strategies to improve the recognition of anal chancres.

Disclosure No significant relationships.

\section{P765 ADDED VALUE OF TREPONEMA PALLIDUM PCR IN DIAGNOSING EARLY SYPHILIS}

${ }^{1}$ Jacky Flipse, ${ }^{2}$ Anne-Marie Niekamp, ${ }^{3}$ Nicole Dukers-Muijrers, ${ }^{4}$ Christian Hoebe, ${ }^{5}$ Petra Wolffs, ${ }^{6}$ Inge Van Loo*. ${ }^{1}$ Isala Hospital, Laboratory for Medical Microbiology and Infectious Diseases, Zwolle, Netherlands; ${ }^{2}$ South Limburg Public Health Service; ${ }^{2}$ Department of Sexual Health, Infectious Diseases and Environmental Health, Heerlen, Netherlands; ${ }^{3}$ Public Health Service South Limburg, Sexual Health, Infectious Diseases and Environmental Health, Heerlen, Netherlands; ${ }^{4}$ Public Health Service South Limburg, Maastricht University Medical Center (MUMC), Sexual Health, Infectious Diseases and Environmental Health Medical Microbiology, Care and Public Health Research Institute (CAPHRI), Heerlen, Netherlands; ${ }^{5}$ Maastricht University Medical Center (MUMC), Medical Microbiology, Care and Public Health Research Institute (CAPHRI), Maastricht, Netherlands; ${ }^{6}$ Maastricht University Medical Center, Medical Microbiology, Maastricht, Netherlands

\subsection{6/sextrans-2019-sti.823}

Background Diagnosing an infection with Treponema pallidum, the causative agent of syphilis, is routinely based on serology. STD clinics routinely screen those with high-risk sexual behaviour, e.g. MSM, for syphilis. In case of an ulcus, swabs taken from the ulcer can be tested for T. pallidum by PCR. Here, we assessed the added value of PCR next to serology in primary syphilis.

Methods Retrospective data were analysed from patients of our STI clinic. Samples were taken from the genital ulcer for 
T. pallidum PCR and syphilis serology was simultaneously performed. Serology was interpreted positive when seroconversion was detected or when RPR significantly increased in those with a history of treated syphilis. Serology was interpreted as negative when the screening was negative or when no significant rise in RPR was detected in those with a history of syphilis.

Results In total 191 PCR - serology combined results were analysed. In total 70/191 (37\%) PCRs were positive. In 24/70 (34\%) samples the positive PCR result added to diagnosing primary syphilis, either because the serology was negative $(n=5,7.1 \%)$ and the diagnosis would have been missed or the positive PCR result added in staging syphilis $(n=19$, $27.0 \%$ ) affecting the treatment regimen. Moreover in 11/76 (14\%) serology positive patients the PCR was negative. Six of these patients were clinically diagnosed as primary syphilis, 3 as syphilis latens recens and 2 as syphilis stage unknown.

Conclusion In our setting, the T. pallidum PCR is of added value in the diagnosis of primary syphilis as without PCR one in 10 early syphilis would have been missed and about one in 5 would have been possibly overtreated. Importantly, the PCR supports the low-threshold testing policy since patients can present within the window period of serology optimizing public health efforts to minimize transmission.

Disclosure No significant relationships.

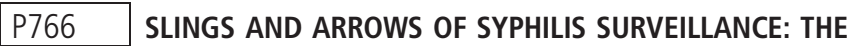 DEPARTMENT OF DEFENSE EXPERIENCE WITH ADMINISTRATIVE CASE FINDING}

${ }^{1}$ Eric Larsen*, ${ }^{2}$ Tony Kim, ${ }^{3}$ Shauna Stahlman, ${ }^{4}$ Anuradha Ganesan, ${ }^{5}$ Eric Garges. ${ }^{1}$ USUHS, Preventive Medicine and Biostatistics, Bethesda, USA; ${ }^{2}$ USUHS, Bethesda, USA; ${ }^{3}$ Defense Health Agency, Armed Forces Health Surveillance Branch, Silver Spring, USA; ${ }^{4}$ Hernry M. Jackson Foundation, Bethesda, USA; ${ }^{5}$ Infectious Disease Clinical Research Program, Department of Preventive Medicine and Biostatistics, Bethesda, USA

10.1136/sextrans-2019-sti.824

Background Routine surveillance of Military Health System (MHS) data provides actionable information on STI rates. National increases in syphilis rates led to the publication of a 2015 MHS syphilis analysis demonstrating a significant rise in military syphilis cases between 2010 and 2015. The current study had two objectives 1) validate the current syphilis estimates under the DoD surveillance case definition and 2) evaluate the validity of clinical staging of syphilis cases within the surveillance period.

Methods Of the initial 2976 incident cases identified in the 2015 surveillance review, we sampled 500 cases. We developed and applied a standardized review algorithm for case determination and expert chart review to provide evidence of clinical stage of disease at the time of surveillance case capture.

Results Out of 500 total cases evaluated, 181 (36\%) were determined to not be cases of syphilis. Surveillance cases identified through Reportable Medical Events (RME) had a positive predictive value (PPV) of 0.82 compared to those cases identified through administratively available (ICD9-coded) data with a PPV of 0.42. Similarly, surveillance classification of clinical staging of infection was grossly inaccurate with respect to Latent, Primary or Secondary (P\&S), or Late infection with accuracy dependent on use of RME (PPV 0.49) vs ICD-9 codes (PPV 0.30) for case identification.
Conclusion A full one third of DoD surveillance case identified cases of syphilis in the Military Health system are not true cases of syphilis. The predominate cause of this misclassification was the reliance on appropriate use of ICDs by providers. The use of administrative data (ICD codes) for incidence and disease stage surveillance should be done with caution due to inappropriate use of coding, misinterpretation of labs, and overly inclusive case definitions. RMEs provide better accuracy (PPV) for correctly identifying incident cases but are still inaccurate with respect to clinical stage.

Disclosure No significant relationships.

\section{P767 SEX, DRUGS AND THE INTERNET - THE PERFECT STORM FOR SYPHILIS TRANSMISSION AMONG BLACK GAY AND BISEXUAL MEN (BMSM)}

1Jacky Jennings*, ${ }^{1}$ Carla Tilchin, ${ }^{2}$ Christina Schumacher, ${ }^{3}$ Matthew Hamill, ${ }^{4}$ Carl Latkin, ${ }^{5}$ Anne Rompalo, ${ }^{6}$ Sebastian Ruhs, ${ }^{7}$ Sarah Rives, ${ }^{5}$ Errol Fields, ${ }^{7}$ Adena Greenbaum, ${ }^{8}$ Khalil Ghanem. 'Johns Hopkins University School of Medicine, Center for Child and Community Health Research (CCHR), Baltimore, USA; ${ }^{2}$ Johns Hopkins School of Medicine, Center for Child and Community Health Research, Baltimore, USA; ${ }^{3}$ Johns Hopkins University, School of Medicine, Baltimore, USA; ${ }^{4}$ Johns Hopkins Bloomberg School of Public Health, Department of Health, Behavior and Society, Baltimore, USA; ${ }^{5}$ Johns Hopkins School of Medicine, Baltimore, USA; ${ }^{6}$ Chase Brexton, Baltimore, USA; ${ }^{7}$ Baltimore City Health Department, Baltimore, USA; ${ }^{8}$ Johns Hopkins Univ MD, USA

10.1136/sextrans-2019-sti.825

Background Use of the internet to meet sex partners has increased over time among MSM, and an outbreak of syphilis has been linked to seeking sex partners through an online chatroom. Other studies have shown associations between methamphetamine use(MU), sexual risk behaviors and STIs including syphilis. The objective was to determine the association between syphilis positivity, MU, sexual risk behaviors and sex partner meeting spaces and separately, MU, sexual risk behaviors and sex partner meeting spaces among Black MSM (BMSM) in one mid-Atlantic U.S. city without a known history of these associations.

Methods Data came from an ongoing longitudinal cohort study, the Understanding Sexual Health in Networks Study (USHINE) including MSM 18-45 years of age in Baltimore City. Information on MU and sex partners and meeting spaces in the past 3 months was obtained via an egocentric sexual network survey. Summary statistics, chi-squared tests and ttests were used for hypothesis testing.

Results 147 MSM were enrolled and 75.5\% (111) were BMSM. The mean age of participants was 29.7 years (SD 5.60); $33.3 \%(34)$ were syphilis positive. Syphilis positivity was associated with MU (57.1\% vs $29.6 \%$, p-value=0.042) and meeting sex partners on Jack'd $(42.3 \%$ vs $23.4 \%$, pvalue $=0.046)$ and Facebook $(52 \%$ vs $27 \%$, p-value $=0.022)$. MU (vs no use) was associated with anonymous sex $(69.2 \%$ vs $19.3 \%$, p-value $<0.000)$, higher numbers of sex partners ( $\geq 3$ in past 3 months $76.9 \%$ vs $25.7 \%$, p-value $<0.001$ ), and meeting sex partners on Jack'd $(84.6 \%$ vs $47.7 \%$, pvalue $=0.012)$, Grindr $(69.2 \%$ vs $25.7 \%$, p-value $<0.001)$, and Adam4Adam (53.9\% vs $15.6 \%$, p-value $<0.001)$.

Conclusion Among BMSM, syphilis infection was associated with MU and specific sex partner meeting spaces, and MU was associated with sexual risk behaviors and specific sex partner meeting spaces. The relationships have not been identified before in this setting and suggest specific meeting spaces may 